\title{
ON COUNTABLY COMPACT 0-SIMPLE TOPOLOGICAL INVERSE SEMIGROUPS
}

\author{
OLEG GUTIK AND DUŠAN REPOVŠ
}

\begin{abstract}
We describe the structure of 0 -simple countably compact topological inverse semigroups and the structure of congruence-free countably compact topological inverse semigroups.
\end{abstract}

We follow the terminology of [3, 4, 8]. In this paper all topological spaces are Hausdorff. If $S$ is a semigroup then we denote the subset of idempotents of $S$ by $E(S)$. A topological space $S$ that is algebraically a semigroup with a continuous semigroup operation is called a topological semigroup. A topological inverse semigroup is a topological semigroup $S$ that is algebraically an inverse semigroup with continuous inversion. If $Y$ is a subspace of a topological space $X$ and $A \subseteq Y$, then we denote by $\operatorname{cl}_{Y}(A)$ the topological closure of $A$ in $Y$.

The bicyclic semigroup $\mathscr{C}(p, q)$ is the semigroup with the identity 1 generated by two elements $p$ and $q$, subject only to the condition $p q=1$. The bicyclic semigroup plays an important role in the algebraic theory of semigroups and in the theory of topological semigroups. For example, the well-known Andersen's result [1] states that a (0-) simple semigroup is completely (0-) simple if and only if it does not contain the bicyclic semigroup. The bicyclic semigroup admits only the discrete topology and a topological semigroup $S$ can contain $\mathscr{C}(p, q)$ only as an open subset [7]. Neither stable nor $\Gamma$-compact topological semigroups can contain a copy of the bicyclic semigroup [2, 12].

Let $S$ be a semigroup and $I_{\lambda}$ a non-empty set of cardinality $\lambda$. We define the semigroup operation ' . ' on the set $B_{\lambda}(S)=I_{\lambda} \times S^{1} \times I_{\lambda} \cup\{0\}$ as follows

$$
(\alpha, a, \beta) \cdot(\gamma, b, \delta)= \begin{cases}(\alpha, a b, \delta), & \text { if } \beta=\gamma \\ 0, & \text { if } \beta \neq \gamma\end{cases}
$$

and $(\alpha, a, \beta) \cdot 0=0 \cdot(\alpha, a, \beta)=0 \cdot 0=0$, for $\alpha, \beta, \gamma, \delta \in I_{\lambda}$, and $a, b \in S^{1}$. The semigroup $B_{\lambda}(S)$ is called a Brandt $\lambda$-extension of the semigroup $S$ [10]. Furthermore, if $A \subseteq S$ then we shall denote $A_{\alpha \beta}=\{(\alpha, s, \beta) \mid s \in A\}$ for $\alpha, \beta \in I_{\lambda}$. If a semigroup $S$ is trivial (i.e. if $S$ contains only one element), then $B_{\lambda}(S)$ is the semigroup of $I_{\lambda} \times I_{\lambda}$-matrix units [4], which we shall denote by $B_{\lambda}$. By Theorem 3.9 of [4, an inverse semigroup $T$ is completely 0 -simple if and only if $T$ is isomorphic to a Brandt $\lambda$-extension $B_{\lambda}(G)$ of some group $G$ and $\lambda \geqslant 1$. We also note that if $\lambda=1$, then the semigroup $B_{\lambda}(S)$ is isomorphic to the semigroup $S$ with adjoint zero. Gutik and Pavlyk [11] proved that any continuous homomorphism from the infinite topological semigroup of matrix units into a compact topological semigroup is annihilating, and hence the infinite topological semigroup of matrix units does not embed into a compact topological semigroup. They also showed that if a topological inverse semigroup $S$ contains a semigroup of matrix units $B_{\lambda}$, then $B_{\lambda}$ is a closed subsemigroup of $S$.

Suschkewitsch [17] proved that any finite semigroup $S$ contains a minimal ideal $K$. He also showed that $K$ is a completely simple semigroup and described the structure of finite simple semigroups. Rees [15] generalized the Suschkewitsch Theorem and showed that if

\footnotetext{
${ }^{0}$ This research was supported by the Slovenian Research Agency grants P1-0292-0101-04 and BI-UA/04-06-007. We thank the referee and the editor for comments.

Date: February 28, 2022.

2000 Mathematics Subject Classification. 20M18, 22A15.

Key words and phrases. Topological inverse semigroup, 0-simple semigroup, completely 0-simple semigroup, Stone-Čech compactification, congruence-free semigroup, bicyclic semigroup, semigroup of matrix units.
} 
a semigroup $S$ contains a minimal ideal $K$ then $K$ is isomorphic to a Rees matrix semigroup $M[G ; I, \Lambda, P]$ over a group $G$ with a regular sandwich matrix $P$. He also proved that any completely 0 -simple semigroup is isomorphic to a Rees matrix semigroup $M[G ; I, \Lambda, P]$ over a 0 -group $G^{0}$ with a regular sandwich matrix $P$. Wallace [18] proved the topological analogue of the Suschkewitsch-Rees Theorem for compact topological semigroups: every compact topological semigroup contains a minimal ideal, which is topologically isomorphic to a topological paragroup. Paalman-de-Miranda [14] proved that any 0-simple compact topological semigroup $S$ is completely 0 -simple, the zero of $S$ is an isolated point in $S$ and $S \backslash\{0\}$ is homeomorphic to the topological product $X \times G \times Y$, where $X$ and $Y$ are compact topological spaces and $G$ is homeomorphic to the underlying space of a maximal subgroup of $S$, contained in $S \backslash\{0\}$. Owen [13] showed that if $S$ a locally compact completely simple topological semigroup, then $S$ has a structure similar to a compact simple topological semigroup. Owen also gave an example which shows that a similar statement does not hold for a locally compact completely 0-simple topological semigroup. Gutik and Pavlyk [11] proved that the subsemigroup of idempotents of a compact 0 -simple topological inverse semigroup is finite, and hence the topological space of a compact 0 -simple topological inverse semigroup is homeomorphic to a finite topological sum of compact topological group and a single point.

A Hausdorff topological space $X$ is called countably compact if any open countable cover of $X$ contains a finite subcover [8]. In this paper we shall prove that the bicyclic semigroup cannot be embedded into any countably compact topological inverse semigroup. We shall also describe the structure of 0-simple countably compact topological inverse semigroups and the structure of congruence-free countably compact topological inverse semigroups.

Theorem 1. A countably compact topological inverse semigroup cannot contain the bicyclic semigroup. Therefore every (0-) simple countably compact topological inverse semigroup is (0-) completely simple.

Proof. Let $T$ be a countably compact topological inverse semigroup and suppose that $T$ contains $\mathscr{C}(p, q)$ as a subsemigroup. Let $S=\mathrm{cl}_{T}(\mathscr{C}(p, q))$. Then by Theorem 3.10.4 of [8], $S$ is a countably compact space and by Proposition II.2 of [7], $S$ is a topological inverse semigroup. Thus by Corollary I.2 of [7], the semigroup $\mathscr{C}(p, q)$ is a discrete subspace of $S$ and by Theorem I.3 of [7, $\mathscr{C}(p, q)$ is an open subspace of $S$ and $S \backslash \mathscr{C}(p, q)$ is an ideal in $S$. Therefore any element of $\mathscr{C}(p, q)$ is an isolated point in the topological space $S$. We define the maps $\varphi: S \rightarrow E(S)$ and $\psi: S \rightarrow E(S)$ by the formulae $\varphi(x)=x x^{-1}$ and $\psi(x)=x^{-1} x$. Since $S \backslash \mathscr{C}(p, q)$ is an ideal of $S, A=\varphi^{-1}(\{1\}) \cup \psi^{-1}(\{1\}) \subseteq \mathscr{C}(p, q)$, and since the maps $\varphi$ and $\psi$ are continuous $A$ is a clopen and hence countably compact infinite subset of $S$. But $A$ is an open subspace of $S$ whose elements are isolated points in $S$. A contradiction.

The second part of the theorem follows from Theorem 2.54 of [4].

Let $\mathscr{S}$ be a class of topological semigroups. Let $\lambda$ be a cardinal $\geqslant 1$, and $(S, \tau) \in \mathscr{S}$. Let $\tau_{B}$ be a topology on $B_{\lambda}(S)$ such that $\left(B_{\lambda}(S), \tau_{B}\right) \in \mathscr{S}$ and $\left.\tau_{B}\right|_{(\alpha, S, \alpha)}=\tau$ for some $\alpha \in I_{\lambda}$. Then $\left(B_{\lambda}(S), \tau_{B}\right)$ is called a topological Brandt $\lambda$-extension of $(S, \tau)$ in $\mathscr{S}$ [10].

Let $\alpha, \beta, \gamma, \delta \in I_{\lambda}$ and $A$ be a subspace of $S$. Since the restriction $\left.\varphi_{\alpha \beta}^{\gamma \delta}\right|_{A_{\alpha \beta}}: A_{\alpha \beta} \rightarrow A_{\gamma \delta}$ of the map $\varphi_{\alpha \beta}^{\gamma \delta}: B_{\lambda}(S) \rightarrow B_{\lambda}(S)$ defined by the formula $\varphi_{\alpha \beta}^{\gamma \delta}(s)=(\gamma, 1, \alpha) \cdot s \cdot(\beta, 1, \delta)$ is a homeomorphism, we get the following:

Lemma 1. Let $\lambda \geqslant 1$ and $B_{\lambda}(S)$ be a topological Brandt $\lambda$-extension of a topological semigroup $S$ and $A$ a subspace of $S$. Then the subspaces $A_{\alpha \beta}$ and $A_{\gamma \delta}$ in $B_{\lambda}(S)$ are homeomorphic for all $\alpha, \beta, \gamma, \delta \in I_{\lambda}$.

Theorem 2. Let $S$ be a 0-simple countably compact topological inverse semigroup. Then there exist a nonempty finite set $I_{\lambda}$ of cardinality $\lambda$ and a countably compact topological group $H$ such that $S$ is topologically isomorphic to a topological Brandt $\lambda$-extension $B_{\lambda}(H)$ of $H$ in the class of topological inverse semigroups. Moreover, $S$ is homeomorphic to a finite topological sum of countable compact topological groups and a single point. 
Proof. By Theorem 1, the semigroup $S$ is completely 0-simple. Now Theorem 3.9 of [4] implies that there exist a nonempty set $I_{\lambda}$ of cardinality $\lambda$ and a group $G$ such that $S$ is algebraically isomorphic to $B_{\lambda}(G)$. Therefore for any $\alpha \in I_{\lambda}$ the subset $G_{\alpha \alpha}$ is a subgroup of $B_{\lambda}(G)$ and since $B_{\lambda}(G)$ is a topological inverse semigroup, a topological subspace $G_{\alpha \alpha}$ of $B_{\lambda}(G)$ with the induced multiplication is a topological group. We fix $\alpha \in I_{\lambda}$ an put $H=G_{\alpha \alpha}$. Then the topological semigroup $S$ is topologically isomorphic to a topological Brandt $\lambda$ extension $B_{\lambda}(H)$ of the topological group $H$.

Let $e_{H}$ be the identity of $H$. Then the subsemigroup $B_{\lambda}\left(e_{H}\right)=\{0\} \cup\left\{\left(\alpha, e_{H}, \beta\right) \mid \alpha, \beta \in I_{\lambda}\right\}$ of $B_{\lambda}(H)$ is algebraically isomorphic to the semigroup of matrix units $B_{\lambda}$. By Theorem 14 [11], $B_{\lambda}\left(e_{H}\right)$ is a closed subsemigroup of $B_{\lambda}(H)$ and hence by Theorem 3.10.4 of [8], $B_{\lambda}\left(e_{H}\right)$ is a countably compact topological space. Therefore Theorem 6 of [11] implies that $B_{\lambda}\left(e_{H}\right)$ is a finite discrete subsemigroup of $B_{\lambda}(H)$ and hence the set $I_{\lambda}$ is finite.

We define the maps $\varphi: B_{\lambda}(H) \rightarrow B_{\lambda}\left(e_{H}\right)$ and $\psi: B_{\lambda}(H) \rightarrow B_{\lambda}\left(e_{H}\right)$ by the formulae $\varphi(x)=$ $x x^{-1}$ and $\psi(x)=x^{-1} x$. Since $B_{\lambda}(H)$ is a topological inverse semigroup the maps $\varphi$ and $\psi$ continuous and hence by Lemma 4 of [11], the set $H_{\alpha \beta}=\varphi^{-1}\left(\left(\alpha, e_{H}, \beta\right)\right) \cap \varphi^{-1}\left(\left(\alpha, e_{H}, \beta\right)\right)$ is clopen in $B_{\lambda}(H)$. By Lemma 1, the subspaces $H_{\alpha \beta}$ and $H_{\gamma \delta}$ are homeomorphic for any $\alpha, \beta, \gamma, \delta \in I_{\lambda}$, and hence all of them are homeomorphic to the topological group $H$.

A Tychonoff topological space $X$ is called pseudocompact if every continuous real-valued function on $X$ is bounded. Since the topological space of $T_{0}$-topological group is Tychonoff and any topological sum of Tychonoff spaces is a Tychonoff space, Theorem 3.10.20 of [8] implies:

Corollary 1. The topological space of a 0-simple countably compact topological inverse semigroup is Tychonoff and hence pseudocompact.

Let $X$ be a topological space. The pair $(Y, c)$, where $Y$ is a compactum and $c: X \rightarrow X$ is a homeomorphic embedding of $X$ into $Y$, such that $\operatorname{cl}_{Y} c(X)=Y$, is called a compactification of the space $X$. Define the ordering $\preccurlyeq$ on the family $\mathcal{C}(X)$ of all compactifications of a topological space $X$ as follows: $c_{2}(X) \preccurlyeq c_{1}(X)$ if and only if there exists a continuous map $f: c_{1}(X) \rightarrow c_{2}(X)$ such that $f c_{1}=c_{2}$. The greatest element of the family $\mathcal{C}(X)$ with respect to the ordering $\preccurlyeq$ is called the Stone-Cech compactification of the space $X$ and it is denoted by $\beta X$. Comfort and Ross [6] proved that the Stone-Cech compactification of a pseudocompact topological group is a topological group. The next theorem is an analogue of the ComfortRoss Theorem:

Theorem 3. Let $S$ be a 0-simple countable compact topological inverse semigroup. Then the Stone-Čech compactification of $S$ admits a structure of 0-simple topological inverse semigroup with respect to which the inclusion mapping of $S$ into $\beta S$ is a topological isomorphism.

Proof. By Theorem 2, $S$ is topologically isomorphic to a Brandt $\lambda$-extension of some topological group $H$ in the class of topological inverse semigroups and $\lambda<\omega$. Now by Lemma 1 , the subspaces $H_{\alpha \beta}$ and $H_{\gamma \delta}$ are homeomorphic in $B_{\lambda}(H)$, for any $\alpha, \beta, \gamma, \delta \in I_{\lambda}$. Since a maximal subgroup in $S$ is closed we have that $H_{\alpha \beta}$ is a clopen subset of $B_{\lambda}(H)$, for every $\alpha, \beta \in I_{\lambda}$. By Corollary 1, the topological space $B_{\lambda}(H)$ is pseudocompact. Since any clopen subspace of a pseudocompact topological space is pseudocompact (see [5]) the subspace $H_{\alpha \beta}$ is pseudocompact, for every $\alpha, \beta \in I_{\lambda}$. Obviously, the topological space $B_{\lambda}(H) \backslash\{0\}$ is homeomorphic to $H \times I_{\lambda} \times I_{\lambda}$. Since the topological space $I_{\lambda} \times I_{\lambda}$ is finite and hence compact, by Corollary 3.10.27 of [8], the space $B_{\lambda}(H) \backslash\{0\}$ is pseudocompact. Now by Theorem 1 of [9], we have $\beta\left(H \times I_{\lambda} \times I_{\lambda}\right)=\beta H \times \beta I_{\lambda} \times \beta I_{\lambda}=\beta H \times I_{\lambda} \times I_{\lambda}$ and therefore $\beta\left(B_{\lambda}(H)\right)=B_{\lambda}(\beta H)$.

Corollary 2. Every 0-simple countable compact topological inverse semigroup is a dense subsemigroup of a 0-simple compact topological inverse semigroup.

If $S$ is completely simple inverse semigroup then the semigroup $S$ with joined zero $S^{0}$ is completely 0 -simple and hence by Theorem 3.9 of [4], the semigroup $S^{0}$ is isomorphic to a Brandt $\lambda$-extension $B_{\lambda}(G)$ of some group $G$. Therefore any nonzero idempotent of $S^{0}$ is 
primitive. Let $e$ and $f$ are nonzero idempotents of $S^{0}$. Since $S$ is an inverse subsemigroup of $S^{0}$ we have $e f=f e \leqslant e$ and $e f=f e \leqslant f$, and hence $e=e f=f$. Thus, the inverse semigroup $S$ contains the unique idempotent and hence it is a group. Therefore a completely simple inverse semigroup is a group and Theorem 1 implies that every simple countable compact topological inverse semigroup is a topological group.

A semigroup $S$ is called congruence-free if it has only two congruences: the identity relation and the universal relation [16].

Theorem 4. Let $S$ be a congruence-free countably compact topological inverse semigroup with zero. Then $S$ is isomorphic to a finite semigroup of matrix units.

Proof. Suppose not. Since the semigroup $S$ contains a zero by Theorem 2, $S$ is topologically isomorphic to a topological Brandt $\lambda$-extension $B_{\lambda}(H)$ of a pseudocompact topological group $H$ in the class of topological inverse semigroups and $\lambda<\omega$. Suppose that the group $H$ is not trivial. Then we define a map $h: B_{\lambda}(H) \rightarrow B_{\lambda}$ by the formulae $h((\alpha, g, \beta))=(\alpha, \beta)$ and $h(0)=0$. Since $h((\alpha, g, \beta)(\gamma, s, \delta))=h((\alpha, g s, \delta))=(\alpha, \delta)=(\alpha, \beta)(\gamma, \delta)=h((\alpha, g, \beta)) h((\gamma, s, \delta))$ for $\beta=\gamma$ and $h((\alpha, g, \beta)(\gamma, s, \delta))=h(0)=0=(\alpha, \beta)(\gamma, \delta)=h((\alpha, g, \beta)) h((\gamma, s, \delta))$ for $\beta \neq \gamma$, the map $h$ is a homomorphism. This contradicts the assumption that $S$ is a congruence-free semigroup.

\section{REFERENCES}

[1] O. Andersen, Ein Bericht über die Struktur abstrakter Halbgruppen, PhD Thesis, Hamburg, 1952.

[2] L. W. Anderson, R. P. Hunter and R. J. Koch, Some results on stability in semigroups. Trans. Amer. Math. Soc. 117 (1965), 521-529.

[3] J. H. Carruth, J. A. Hildebrant and R. J. Koch, The Theory of Topological Semigroups, I, II. Marcel Dekker, Inc., New York and Basel, 1983 and 1986.

[4] A. H. Clifford and G. B. Preston, The Algebraic Theory of Semigroups, I, II. Amer. Math. Soc., Providence, R.I. 1961 and 1967.

[5] J. Colmex, Sur les espaces precompacts, C. R. Acad. Sci. Paris 233 (1951), 1552-1553.

[6] W. W. Comfort and K. A. Ross, Pseudocompactness and uniform continuity in topological groups, Pacif. J. Math. 16 (1966), 483-496.

[7] C. Eberhart and J. Selden, On the closure of the bicyclic semigroup, Trans. Amer. Math. Soc. 144 (1969), 115-126.

[8] R. Engelking, General Topology, Second Ed. PWN, Warsaw, 1986.

[9] I. Glicksberg, Stone-Čech compactifications of products, Trans. Amer. Math. Soc. 90 (1959), 369-382.

[10] O. V. Gutik and K. P. Pavlyk, H-closed topological semigroups and topological Brandt $\lambda$-extensions, Math. Methods and Phys.-Mech. Fields 44:3 (2001), 20-28. (in Ukrainian)

[11] O. V. Gutik and K. P. Pavlyk, On topological semigroups of matrix units, Semigroup Forum 71 (2005), $389-400$.

[12] J. A. Hildebrant and R. J. Koch, Swelling actions of $\Gamma$-compact semigroups, Semigroup Forum 33 (1988), $65-85$.

[13] W. S. Owen, The Rees theorem for locally compact semigroups, Semigroup Forum 6 (1973), 133-152.

[14] A. B. Paalman-de-Miranda, Topological Semigroup, Mathematical Centre Tracts. Vol. 11. Mathematisch Centrum, Amsterdam, 1964.

[15] D. Rees, On semi-groups, Proc. Cambridge Phil. Soc. 36 (1940), 387-400.

[16] B. M. Schein, Homomorphisms and subdirect decompositions of semigroups, Pacif. J. Math. 24 (1966), $529-547$.

[17] A. Suschkewitsch, Uber die endlichen Gruppen, Math. Ann. 99 (1928), 529 - 547.

[18] A. D. Wallace, The Suschkewitsch-Rees structure theorem for compact simple semigroups, Proc. Nat. Acad. Sci. 42 (1956), 430-432.

Department of Mathematics, Ivan Franko Lviv National University, Universytetska 1, LVIV, 79000, UKRAINE

E-mail address: o_gutik@franko.lviv.ua, ovgutik@yahoo.com

Institute of Mathematics, Physics and Mechanics, and Faculty of Education, University of Luubluana, P.O.Box 2964, Ljubljana, 1001, Slovenia

E-mail address: dusan.repovs@guest.arnes.si 